TRANSACTIONS OF THE

AMERICAN MATHEMATICAL SOCIETY

Volume 364, Number 2, February 2012, Pages 721-744

S 0002-9947(2011)05313-4

Article electronically published on October 5, 2011

\title{
NONCOMMUTATIVE SEMIALGEBRAIC SETS AND ASSOCIATED LIFTING PROBLEMS
}

\author{
TERRY A. LORING AND TATIANA SHULMAN
}

\begin{abstract}
We solve a class of lifting problems involving approximate polynomial relations (soft polynomial relations). Various associated $C^{*}$-algebras are therefore projective. The technical lemma we need is a new manifestation of Akemann and Pedersen's discovery of the norm adjusting power of quasi-central approximate units.

A projective $C^{*}$-algebra is the analog of an absolute retract. Thus we can say that various noncommutative semialgebraic sets turn out to be absolute retracts. In particular we show that a noncommutative absolute retract results from the intersection of the approximate locus of a noncommutative homogeneous polynomial with the noncommutative unit ball. By unit ball we are referring to the $C^{*}$-algebra of the universal row contraction. We show that various alternative noncommutative unit balls are also projective.

Sufficiently many $C^{*}$-algebras are now known to be projective so that we are able to show that the cone over any separable $C^{*}$-algebra is the inductive limit of $C^{*}$-algebras that are projective.
\end{abstract}

\section{INTRODUCTION}

Lifting problems for relations in $C^{*}$-algebras have tended to have ad hoc solutions. Olsen and Pedersen prove in 23] that a nilpotent always has a nilpotent lift, specifically that given $x$ in a $C^{*}$-algebra quotient $A / I$ with

$$
x^{n}=0
$$

there is always $X$ in $A$ with $\pi(X)=x$ and $X^{n}=0$. Their proof is rather different from the techniques Akemann and Pedersen used in 2] to show that for $x$ in $A / I$ with

$$
\left\|x^{n}\right\| \leq \epsilon \quad(\epsilon>0)
$$

there is always a lift $X$ with $\left\|X^{n}\right\| \leq \epsilon$. Different still are the techniques used in 22 to show that the relations describing $\mathbf{C M}_{n}=C_{0}\left((0,1], \mathbf{M}_{n}\right)$ are liftable: given

Received by the editors August 23, 2009 and, in revised form, January 29, 2010 and February $10,2010$.

2010 Mathematics Subject Classification. Primary 46L05, 47 B99.

Key words and phrases. $C^{*}$-algebra, relation, projectivity, row contraction, noncommutative star-polynomial, lifting.

(C)2011 American Mathematical Society Reverts to public domain 28 years from publication 
$x_{1}, \ldots, x_{n}$ in $A / I$ satisfying all the relations

$$
\begin{aligned}
\left\|x_{j}\right\| & \leq 1 \quad(\forall j), \\
x_{j}^{*} x_{k} & =0 \quad(j \neq k), \\
x_{j}^{*} x_{j} & =x_{k}^{*} x_{k} \quad(\forall j, \forall k), \\
x_{1} x_{1}^{*} & =x_{1}^{*} x_{1},
\end{aligned}
$$

there are lifts $X_{1}, \ldots, X_{n}$ in $A$ that also satisfy these relations. More recently, $M$-ideals showed up in 24] to settle the lifting problem for the relations

$$
\begin{aligned}
\|x\| & \leq 1, \\
x^{n} & =0 .
\end{aligned}
$$

The lifting results above (most of them, anyway) show various $C^{*}$-algebras are projective. Projectivity was introduced by Effros and Kaminker in [10. A $C^{*}$ algebra $P$ is projective if the map

$$
\rho \circ-: \operatorname{hom}(P, B) \rightarrow \operatorname{hom}(P, C)
$$

is onto whenever $\rho: B \rightarrow C$ is onto.

Projectivity was shown by Blackadar in 4 to be the noncommutative analog of a space being an absolute retract (AR). The analog of absolute neighborhood retract (ANR) is semiprojectivity, which we will not discuss in detail in this paper except in Section 6 .

Systematic investigations of projectivity exist, but only in the case of at most one-dimensional spectrum. There was a study of $C_{0}(X)$ for $X^{+}$a tree in [17. In [7, Chigogidze and Dranishnikov solved the general question for $C_{0}(X)$ being projective. The answer is that $C_{0}(X)$ is projective if and only if $X^{+}$is a dendrite. The finite mapping telescopes associated to inclusions of finite-dimensional $C^{*}$ algebras were shown to be projective in [22]. In the later terminology of [13], this says we have projectivity for a large class of one-dimensional noncommutative $\mathrm{CW}$ complexes.

"NC" will stand for "noncommutative." Thus noncommutative CW complex becomes NCCW.

In the commutative case, very sweeping statements can be made about what spaces are AR or ANR. For example, every compact semialgebraic set in finitedimensional Euclidean space is an absolute neighborhood retract; see [25, p. 79] and [16] for precise results and definitions. A subset of Euclidean space is said to be semialgebraic if it is the union of solution sets of polynomial equations and polynomial inequalities. As we are interested in closed and connected sets, it will suffice to have in mind sets of the form

$$
\left\{\left(x_{1}, \ldots, x_{n}\right) \in \mathbb{R}^{n} \mid p_{j}\left(x_{1}, \ldots, x_{n}\right) \leq \epsilon_{j} \text { for } j=1 \ldots J\right\},
$$

where the $p_{j}$ are polynomials.

This general result about semialgebraic sets being ANR cannot translate directly to $C^{*}$-algebras. We know that for the unit disk $\mathbb{D}$, the $C^{*}$-algebra

$$
C_{0}(\mathbb{D} \backslash\{0\}) \cong C^{*}\left\langle x \mid x^{*} x=x x^{*},\|x\| \leq 1\right\rangle
$$

fails to be projective. Normals do not generally lift to normals. Some normals fail to have partial lifts and are bounded away from other normals that have partial lifts. To get technical, $C_{0}(\mathbb{D} \backslash\{0\})$ is not even weakly semiprojective $\left.(12]\right)$. 
TABLE 1. Some known projective $C^{*}$-algebras / liftable relations

\begin{tabular}{|c|c|c|c|c|}
\hline $\begin{array}{l}\text { Gen- } \\
\text { erators }\end{array}$ & $\begin{array}{l}\text { Individual } \\
\text { restrictions }\end{array}$ & Other Relations & $\begin{array}{l}\text { Name or } \\
\text { Comment }\end{array}$ & Credit \\
\hline$x$ & $\begin{array}{l}\|x\| \leq 1 \\
x^{n}=0\end{array}$ & & & $\begin{array}{l}\text { Shulman }[24] \\
\text { (Olsen, } \\
\text { Pedersen, } \\
\text { Loring for } \\
\|x\|<1 .) \\
\end{array}$ \\
\hline$x$ & $\begin{array}{l}\|x\| \leq 1 \\
\left\|x^{n}\right\| \leq C\end{array}$ & & & $\begin{array}{l}\text { Akemann, } \\
\text { Pedersen [1] }\end{array}$ \\
\hline$x, y$ & $\begin{array}{l}\|x\| \leq 1 \\
\|y\| \leq 1\end{array}$ & $\begin{array}{l}x^{*} x=y^{*} y \\
x^{*} y=y^{*} x=0 \\
x^{2}=y^{2}=0\end{array}$ & $C_{0}\left((0,1], \mathbf{M}_{3}\right)$ & $\begin{array}{c}\text { Loring, } \\
\text { Pedersen [22] }\end{array}$ \\
\hline$x, y$ & $\begin{array}{l}-1 \leq x \leq 1 \\
-1 \leq y \leq 1\end{array}$ & $x y=0$ & $C_{0}\left((0,1], \mathbb{C}^{4}\right)$ & Loring [17] \\
\hline$x_{1}, \ldots, x_{n}$ & $\left\|x_{j}\right\| \leq 1$ & $\begin{array}{l}x_{i}^{*} x_{i}=x_{j}^{*} x_{j} \quad(\forall i, \forall j) \\
\left.x_{i}^{*} x_{j}=0 \quad \text { (if } i \neq j\right)\end{array}$ & & $\begin{array}{c}\text { Loring, } \\
\text { Pedersen [22, } \\
\text { Example 3.11] }\end{array}$ \\
\hline$h, k, x$ & & $\begin{array}{l}h k=0 \\
0 \leq\left[\begin{array}{cc}1-h & x^{*} \\
x & k\end{array}\right] \leq 1\end{array}$ & $\begin{array}{l}\text { Useful in the } \\
q \mathbb{C} \text { picture of } \\
K \text {-theory. }\end{array}$ & Loring [19] \\
\hline$x, y, z, w$ & $\begin{array}{l}0 \leq x \leq 1 \\
0 \leq y \leq 1 \\
0 \leq z \leq 1 \\
0 \leq w \leq 1\end{array}$ & $\begin{array}{l}x y=0 \\
z w=0 \\
(1-x) z(1-x)=0 \\
(1-x) w(1-x)=0\end{array}$ & $\begin{array}{l}C_{0}(X) \text { where } \\
X^{+} \text {is a tree } \\
\text { with four } \\
\text { edges. }\end{array}$ & Loring 17. \\
\hline$x_{1}, \ldots, x_{r}$ & & $\left\|\sum_{k=1}^{r} x_{k} x_{k}^{*}\right\| \leq 1$ & & $\begin{array}{l}\text { Folklore, } \\
\text { functional } \\
\text { calculus }\end{array}$ \\
\hline
\end{tabular}

There is a way to avoid the difficulty posed by this nonliftable example other than keeping to small dimension. We will avoid exact relations.

An important instance of Theorem 3.2 is the fact that for any positive $\epsilon$, an element $x$ in a $C^{*}$-algebra quotient $A / I$ with

$$
\begin{aligned}
\|x\| & \leq 1, \\
\left\|x^{*} x-x x^{*}\right\| & \leq \epsilon
\end{aligned}
$$

has a lift to $X$, so $\pi(X)=x$, with $\|X\| \leq 1$ and $\left\|X^{*} X-X X^{*}\right\| \leq \epsilon$. Put another way, we show

$$
A_{\epsilon}=C^{*}\left\langle x \mid\left\|x^{*} x-x x^{*}\right\| \leq \epsilon,\|x\| \leq 1\right\rangle
$$

is projective for all positive $\epsilon$. Since

$$
C_{0}(\mathbb{D} \backslash\{0\}) \cong \lim _{\rightarrow} A_{\frac{1}{k}},
$$

we have shown $C_{0}(\mathbb{D} \backslash\{0\})$ has a shape system (cf. [4, 6, 10]) that is trivial in the sense that all the $C^{*}$-algebras in the system are projective. It was previously unknown whether $C_{0}(\mathbb{D} \backslash\{0\})$ could be written as an inductive limit of semiprojective $C^{*}$-algebras. 
TABLE 2. Some new projective $C^{*}$-algebras/liftable relations

\begin{tabular}{|c|c|c|c|c|}
\hline $\begin{array}{l}\text { Gen- } \\
\text { erators }\end{array}$ & $\begin{array}{l}\text { Individual } \\
\text { restrictions }\end{array}$ & Other Relations & $\begin{array}{l}\text { Name / } \\
\text { Remark }\end{array}$ & Reference \\
\hline$h, k$ & $\begin{array}{l}-1 \leq h \leq 1 \\
-1 \leq k \leq 1\end{array}$ & $\|[h, k]\| \leq C$ & Soft Square & Theorem 3.1 \\
\hline$h, k$ & $\begin{array}{l}-1 \leq h \leq 1 \\
-1 \leq k \leq 1\end{array}$ & $\begin{array}{l}\|[h, k]\| \leq C \\
\|h+i k\| \leq 1\end{array}$ & Soft Disk I & Theorem 3.1 \\
\hline$x$ & $\|x\| \leq 1$ & $\left\|\left[x^{*}, x\right]\right\| \leq 2 C$ & $\begin{array}{l}\text { Soft Disk I } \\
\text { a second } \\
\text { presentation }\end{array}$ & Theorem 3.1 \\
\hline$h, k$ & $\begin{array}{l}-1 \leq h \leq 1 \\
-1 \leq k \leq 1\end{array}$ & $\begin{array}{l}\|[h, k]\| \leq C \\
\left\|h^{2}+k^{2}\right\| \leq 1\end{array}$ & Soft Disk II & Theorem 3.1 \\
\hline$a, k$ & $\begin{array}{l}\|a\| \leq 1 \\
0 \leq k \leq 1\end{array}$ & $\|[a, k]\| \leq C$ & $\begin{array}{l}\text { Might be } \\
\text { useful inves- } \\
\text { tigating } \\
\text { commuta- } \\
\text { tors and } \\
\text { square roots }\end{array}$ & Theorem 3.1 \\
\hline$h, k, x$ & & $\begin{array}{l}\|h k\| \leq C \\
0 \leq\left[\begin{array}{cc}1-h & x^{*} \\
x & k\end{array}\right] \leq 1\end{array}$ & & Theorem 3.1 \\
\hline$x, y, z, w$ & $\begin{array}{l}0 \leq x \leq 1 \\
0 \leq y \leq 1 \\
0 \leq z \leq 1 \\
0 \leq w \leq 1\end{array}$ & $\begin{array}{l}\|x y\| \leq C \\
\|z w\| \leq C \\
\|(1-x) z(1-x)\| \leq C \\
\|(1-x) w(1-x)\| \leq C\end{array}$ & & Theorem 4.2 \\
\hline$x_{1}, \ldots, x_{r}$ & & $\left\|\sum_{k=1}^{r}\left(x_{k} x_{k}^{*}\right)^{\frac{q}{2}}\right\| \leq 1$ & $1<q<\infty$ & Theorem 5.3 \\
\hline
\end{tabular}

An important special case that we study is the approximate zero locus of a homogeneous $\mathrm{NC} *$-polynomial intersected with the $\mathrm{NC}$ unit ball. The homogeneity is imposed to give contractability, and so gives us an expectation of finding not only semiprojectivity, but projectivity. By approximate zero locus we mean the universal $C^{*}$-algebra

$$
A_{\epsilon}=C^{*}\left\langle x_{1}, \ldots, x_{r} \mid\left\|p\left(x_{1}, \ldots, x_{r}\right)\right\| \leq \epsilon,\left\|\sum_{j=1}^{r} x_{j} x_{j}^{*}\right\| \leq 1\right\rangle,
$$

and the "row contraction" condition $\sum x_{j} x_{j}^{*} \leq 1$ (cf. 3, 9]) is implementing the intersection with the unit ball. A special case of Theorem 3.2 states that $A_{\epsilon}$ is projective for all $\epsilon>0$.

In many cases the relations we can handle have as their universal $C^{*}$-algebra something that is very unfamiliar. In these cases, it is perhaps best to see our results as lifting results for the relations rather than projectivity results for the $C^{*}$-algebras. 
Table 1 lists some known liftable relations. It is somewhat representative. There are closure results, such as when $A$ is projective $\mathbf{M}_{n}(A)$ is also projective, which leads to many more liftable sets of relations, so no table can be complete.

Table 2 lists some of the new examples. It is not certain these are new projective $C^{*}$-algebras, as projective $C^{*}$-algebras are contractible and so invariants such as $K$-theory are of no avail. We can say with some certainty the relations were not known to lift.

A useful result in topological shape theory is that every compact metric space is the projective limit of ANRs [6, IX.1.4]. Blackadar writes in [4, 4.4]:

It is not clear that every $\mathrm{C}^{*}$-algebra has a strong shape system [is an inductive limit of semiprojective $\mathrm{C}^{*}$-algebras].

We show in Section 7 that every cone over a separable $C^{*}$-algebra is the inductive limit of projective $C^{*}$-algebras.

\section{QuAsi-CEntral aPPROXimate Units FiX NORMS}

Our key tool for lifting is Theorem 2.3. It was extracted from the difficult terrain that is page 127 of Akemann and Pedersen's paper 2 .

Approximate units are assumed to satisfy $0 \leq u_{\lambda} \leq 1$. If $I$ is an ideal in $A$, we let $\pi: A \rightarrow A / I$ denote the quotient map.

Lemma 2.1. Suppose $I \triangleleft A$. For any approximate unit $u_{\lambda}$ of $I$, any $h$ in $A_{+}$, and any real $0 \leq \delta \leq 1$,

$$
\underset{\lambda}{\limsup }\left\|\left(1-u_{\lambda}\right)^{\frac{1}{2}} h\left(1-u_{\lambda}\right)^{\frac{1}{2}}+(1-\delta) u_{\lambda}^{\frac{1}{2}} h u_{\lambda}^{\frac{1}{2}}\right\| \leq \max (\|\pi(h)\|,(1-\delta)\|h\|) .
$$

Proof. We can lift $\pi(h)$ to $k$ with $0 \leq k \leq\|\pi(h)\|$. Setting $x=h-k$, we have $x$ in $I$ and

$$
\begin{aligned}
& \underset{\lambda}{\limsup }\left\|\left(1-u_{\lambda}\right)^{\frac{1}{2}} h\left(1-u_{\lambda}\right)^{\frac{1}{2}}+(1-\delta) u_{\lambda}^{\frac{1}{2}} h u_{\lambda}^{\frac{1}{2}}\right\| \\
&=\limsup _{\lambda}\left\|\left(1-u_{\lambda}\right)^{\frac{1}{2}} k\left(1-u_{\lambda}\right)^{\frac{1}{2}}+(1-\delta) u_{\lambda}^{\frac{1}{2}} h u_{\lambda}^{\frac{1}{2}}\right\| .
\end{aligned}
$$

Now we use the order structure in $A$ and find

$$
\begin{aligned}
\left(1-u_{\lambda}\right)^{\frac{1}{2}} k\left(1-u_{\lambda}\right)^{\frac{1}{2}}+(1-\delta) u_{\lambda}^{\frac{1}{2}} h u_{\lambda}^{\frac{1}{2}} & \leq\|k\|\left(1-u_{\lambda}\right)+(1-\delta)\|h\| u_{\lambda} \\
& \leq \max (\|\pi(h)\|,(1-\delta)\|h\|) .
\end{aligned}
$$

Lemma 2.2. Suppose $I \triangleleft A$. For any approximate unit $u_{\lambda}$ of $I$ that is quasi-central for $A$, any $a$ in $A$, and any real $0 \leq \delta \leq 1$,

$$
\limsup _{\lambda}\left\|a\left(1-\delta u_{\lambda}\right)^{\frac{1}{2}}\right\| \leq \max \left(\|\pi(a)\|,(1-\delta)^{\frac{1}{2}}\|a\|\right)
$$


Proof. Using the quasi-central property and Lemma 2.1

$$
\begin{aligned}
\underset{\lambda}{\limsup \left\|a\left(1-\delta u_{\lambda}\right)^{\frac{1}{2}}\right\|^{2}} & =\underset{\lambda}{\limsup }\left\|a^{*} a\left(1-\delta u_{\lambda}\right)\right\| \\
& =\underset{\lambda}{\limsup }\left\|a^{*} a\left(1-u_{\lambda}\right)+(1-\delta) a^{*} a u_{\lambda}\right\| \\
& =\underset{\lambda}{\limsup }\left\|\left(1-u_{\lambda}\right)^{\frac{1}{2}} a^{*} a\left(1-u_{\lambda}\right)^{\frac{1}{2}}+(1-\delta) u_{\lambda}^{\frac{1}{2}} a^{*} a u_{\lambda}^{\frac{1}{2}}\right\| \\
& \leq \max \left(\left\|\pi\left(a^{*} a\right)\right\|,(1-\delta)\left\|a^{*} a\right\|\right) \\
& =(\max (\|\pi(a)\|,(1-\delta)\|a\|))^{2} .
\end{aligned}
$$

Theorem 2.3. Suppose $I \triangleleft A$, that $u_{\lambda}$ is a approximate unit $u_{\lambda}$ for $I$ that is quasi-central for $A$, and $a$ is in $A$. If $f$ is a continuous function on $[0,1]$ so that

$$
1=f(0) \geq f(t) \geq f(1) \geq 0,
$$

then

$$
\limsup \left\|a f\left(u_{\lambda}\right)\right\| \leq \max (\|\pi(a)\|, f(1)\|a\|) .
$$

Proof. Let $\delta=1-f(1)^{2}$ and

$$
g(t)=\delta^{-1}\left(1-f(t)^{2}\right) .
$$

This function is continuous and

$$
0=g(0) \leq g(t) \leq g(1)=1,
$$

so $g\left(u_{\lambda}\right)$ is also a quasi-central approximate unit. By Lemma 2.2 ,

$$
\begin{aligned}
\underset{\lambda}{\limsup \left\|a f\left(u_{\lambda}\right)\right\|} & =\underset{\lambda}{\limsup }\left\|a\left(1-\delta g\left(u_{\lambda}\right)\right)^{\frac{1}{2}}\right\| \\
& \leq \max \left(\|\pi(a)\|,(1-\delta)^{\frac{1}{2}}\|a\|\right) \\
& =\max (\|\pi(a)\|, f(1)\|a\|) .
\end{aligned}
$$

\section{LifTing SOFTENED HOMOGENEOUS RELATIONS}

We will consider $*$-polynomials in infinitely many variables that are homogeneous in some finite subset of the variables. These we take to be the first $r$-variables, which we label $x_{1}, \ldots, x_{r}$, and the remaining variables we label $y_{1}, y_{2}, \ldots$ We also use the $n$-tuple notation $\mathbf{x}=\left(x_{1}, \ldots, x_{r}\right)$ and $\mathbf{y}=\left(y_{1}, y_{2}, \ldots\right)$, and with an NC *-polynomial $p$ we use the notation

$$
p(\mathbf{x}, \mathbf{y})=p\left(x_{1}, \ldots, x_{r}, y_{1}, y_{2}, \ldots\right) .
$$

For scalar $t$ we use

$$
t \mathbf{x}=\left(t x_{1}, \ldots, t x_{r}\right) .
$$

We will say $p$ is $d$-homogeneous in the first $r$ variables if

$$
p(t \mathbf{x}, \mathbf{y})=t^{d} p(\mathbf{x}, \mathbf{y})
$$

for all real scalars $t$. In other words, in each monomial the $x_{j}$ and $x_{j}^{*}$ appear collectively $d$ times. As $d$ is not necessarily the degree of $p$, we call $d$ the degree of homogeneity of $p$. 
We will rather quickly run out of letters if we insist on other symbols when evaluating $p$ on specific elements of a $C^{*}$-algebra $A$. Given $x_{1}, \ldots, x_{r}$ in $A$ and $m$ in $A$ or $\widetilde{A}$ we define

$$
m \mathbf{x}=\left(m x_{1}, \ldots, m x_{r}\right) .
$$

(We use $\widetilde{A}$ to denote the unitization of $A$.) If $\varphi: A \rightarrow B$, then

$$
\varphi(\mathbf{x})=\left(\varphi\left(x_{1}\right), \ldots, \varphi\left(x_{r}\right)\right)
$$

and

$$
\varphi(\mathbf{y})=\left(\varphi\left(y_{1}\right), \varphi\left(y_{2}\right), \ldots\right) .
$$

If $z_{1}, \ldots, z_{r}$ are in $A$, then $\mathbf{z} \leq \mathbf{y}$ shall mean $z_{j} \leq x_{j}$ for $j=1, \ldots, r$. If $\mathbf{0} \leq \mathbf{x}$, then $\mathbf{x}^{\frac{1}{2}}$ shall denote $\left(x_{1}^{\frac{1}{2}}, \ldots, x_{r}^{\frac{1}{2}}\right)$. For $a_{1}, \ldots, a_{n}$ and $b_{1}, \ldots, b_{n}$ in $A$, we use the notation

$$
a \bullet b=a_{1} b_{1}+\cdots+a_{n} b_{n} .
$$

Recall $\pi$ is our generic notation for the quotient map $A \rightarrow A / I$.

Theorem 3.1. Suppose $p_{1}, \ldots, p_{J}$ are $N C$ *-polynomials in infinitely many variables that are homogeneous in the first $r$ variables, with each degree of homogeneity $d_{j}$ at least one. Suppose $C_{j}>0$ are constants. For every $C^{*}$-algebra $A$ and $I \triangleleft A$ an ideal, given $x_{1}, \ldots, x_{r}$ and $y_{1}, y_{2}, \ldots$ in $A$ with $\mathbf{0} \leq \mathbf{x}$ and

$$
\left\|p_{j}(\pi(\mathbf{x}), \pi(\mathbf{y}))\right\| \leq C_{j},
$$

there are $z_{1}, \ldots, z_{r}$ in $A$ with $\mathbf{0} \leq \mathbf{z} \leq \mathbf{x}$ and $\pi(\mathbf{z})=\pi(\mathbf{x})$ and

$$
\left\|p_{j}(\mathbf{z}, \mathbf{y})\right\| \leq C_{j} \text {. }
$$

Proof. Our proof is modeled on that of [2, Theorem 3.7].

We start by performing the easier lifting where $C_{j}$ in (3.1) is replaced by $\left(1+\epsilon_{1}\right) C_{j}$. We pick $\epsilon_{1}$ later, but it will be positive. Since $C_{j}$ is not allowed to be zero, $\left(1+\epsilon_{1}\right) C_{j}$ will be strictly larger than $C_{j}$.

Let $u_{\lambda}$ be any approximate unit $u_{\lambda}$ for $I$ that is quasi-central for $A$. By quasicentrality and the homogeneity in $\mathbf{x}$, we have

$$
\begin{aligned}
\lim _{\lambda}\left\|p_{j}\left(\mathbf{x}^{\frac{1}{2}} \bullet\left(1-u_{\lambda}\right) \mathbf{x}^{\frac{1}{2}}, \mathbf{y}\right)\right\| & =\lim _{\lambda}\left\|p_{j}(\mathbf{x}, \mathbf{y})\left(1-u_{\lambda}\right)^{d_{j}}\right\| \\
& \leq \lim _{\lambda}\left\|p_{j}(\mathbf{x}, \mathbf{y})\left(1-u_{\lambda}\right)\right\| \\
& =\left\|\pi\left(p_{j}(\mathbf{x}, \mathbf{y})\right)\right\| \\
& =\left\|p_{j}(\pi(\mathbf{x}), \pi(\mathbf{y}))\right\| \\
& \leq C_{j} .
\end{aligned}
$$

We define

$$
z_{k}^{(1)}=x_{k}^{\frac{1}{2}}\left(1-u_{\lambda_{1}}\right) x_{k}^{\frac{1}{2}},
$$

where $\lambda_{1}$ is large enough to give us

$$
\left\|p_{j}\left(\mathbf{z}^{(1)}, \mathbf{y}\right)\right\| \leq\left(1+\epsilon_{1}\right) C_{j}
$$

for $j=1, \ldots, J$. Clearly, $0 \leq \mathbf{z}^{(1)} \leq \mathbf{x}$ and $\pi\left(\mathbf{z}^{(1)}\right)=\pi(\mathbf{x})$.

We will create ever better lifts by defining

$$
z_{k}^{(2)}=\left(z_{k}^{(1)}\right)^{\frac{1}{2}}\left(1-\delta_{2} u_{\lambda_{2}}\right)\left(z_{k}^{(1)}\right)^{\frac{1}{2}},
$$


and so forth. For consistency, we let $\delta_{1}=1$ and $\mathbf{z}^{(0)}=\mathbf{x}$. We choose $\delta_{1}>\delta_{2}>\cdots$ all positive with

$$
\sum_{c=1}^{\infty} \delta_{c}<\infty
$$

We set $\epsilon_{1}$ and the rest of a sequence $\epsilon_{c}$ by requiring

$$
\left(1-\delta_{c+1}\right)\left(1+\epsilon_{c}\right)=1 .
$$

Notice the $\epsilon_{c}$ are positive and decreasing to zero.

Assume we have found $\mathbf{z}^{(1)}$ through $\mathbf{z}^{(c-1)}$ with

$$
\begin{gathered}
\mathbf{0} \leq \mathbf{z}^{(c-1)} \leq \mathbf{z}^{(c-2)} \cdots \leq \mathbf{z}^{(1)} \leq \mathbf{x} \\
\pi\left(\mathbf{z}^{(c-1)}\right)=\pi\left(\mathbf{z}^{(c-2)}\right)=\cdots=\pi\left(\mathbf{z}^{(1)}\right)=\pi(\mathbf{x}),
\end{gathered}
$$

and

$$
\left\|p_{j}\left(\mathbf{z}^{(w)}, \mathbf{y}\right)\right\| \leq\left(1+\epsilon_{w}\right) C_{j}, \quad(w=1, \ldots, c-1) .
$$

Moreover, assume the $\mathbf{z}^{(w)}$ have been constructed via the formula

$$
\mathbf{z}^{(w)}=\left(\mathbf{z}^{(w-1)}\right)^{\frac{1}{2}}\left(1-\delta_{w} u_{\lambda_{w}}\right)\left(\mathbf{z}^{(w-1)}\right)^{\frac{1}{2}}, \quad(w=1, \ldots, c-1) .
$$

Theorem 2.3 tells us

$$
\begin{aligned}
\underset{\lambda}{\limsup }\left\|p_{j}\left(\left(\mathbf{z}^{(c-1)}\right)^{\frac{1}{2}} \bullet\left(1-\delta_{c} u_{\lambda}\right)\left(\mathbf{z}^{(c-1)}\right)^{\frac{1}{2}}, \mathbf{y}\right)\right\| \\
\quad=\limsup _{\lambda}\left\|p_{j}\left(\mathbf{z}^{(c-1)}, \mathbf{y}\right)\left(1-\delta_{c} u_{\lambda}\right)^{d_{j}}\right\| \\
\leq \limsup _{\lambda}\left\|p_{j}\left(\mathbf{z}^{(c-1)}, \mathbf{y}\right)\left(1-\delta_{c} u_{\lambda}\right)\right\| \\
\leq \max \left(\left\|\pi\left(p_{j}\left(\mathbf{z}^{(c-1)}, \mathbf{y}\right)\right)\right\|,\left(1-\delta_{c}\right)\left\|p_{j}\left(\mathbf{z}^{(c-1)}, \mathbf{y}\right)\right\|\right) \\
=\max \left(\left\|p_{j}(\pi(\mathbf{x}), \pi(\mathbf{y}))\right\|,\left(1-\delta_{c}\right)\left\|p_{j}\left(\mathbf{z}^{(c-1)}, \mathbf{y}\right)\right\|\right) \\
\leq \max \left(C_{j},\left(1-\delta_{c}\right)\left(\left(1+\epsilon_{c-1}\right) C_{j}\right)\right) \\
=C_{j},
\end{aligned}
$$

so we may choose $\lambda_{c}$ with

$$
\left\|p_{j}\left(\left(\mathbf{z}^{(c-1)}\right)^{\frac{1}{2}} \bullet\left(1-\delta_{c} u_{\lambda_{c}}\right)\left(\mathbf{z}^{(c-1)}\right)^{\frac{1}{2}}, \mathbf{y}\right)\right\| \leq\left(1+\epsilon_{c}\right) C_{j} .
$$

We set

$$
\mathbf{z}^{(c)}=\left(\mathbf{z}^{(c-1)}\right)^{\frac{1}{2}}\left(1-\delta_{c} u_{\lambda_{c}}\right)\left(\mathbf{z}^{(c-1)}\right)^{\frac{1}{2}},
$$

and the construction continues.

We wish to set $z_{k}=\lim _{c} z_{j}^{(c)}$, and we may because

$$
\begin{aligned}
\left\|z_{k}^{(c)}-z_{k}^{(c-1)}\right\| & =\left\|\left(z_{k}^{(c-1)}\right)^{\frac{1}{2}}\left(\delta_{c} u_{\lambda_{c}}\right)\left(z_{k}^{(c-1)}\right)^{\frac{1}{2}}\right\| \\
& \leq\left\|z_{k}^{(c-1)}\right\|\left\|\delta_{c} u_{\lambda_{c}}\right\| \\
& \leq 2\left\|x_{k}\right\| \delta_{c} .
\end{aligned}
$$


Equations (3.3), (3.4), and (3.5) give us $\mathbf{0} \leq \mathbf{z} \leq \mathbf{x}$ and $\pi(\mathbf{z})=\mathbf{x}$ and finally the norm conditions

$$
\left\|p_{j}(\mathbf{z}, \mathbf{y})\right\|=\lim _{c}\left\|p_{j}\left(\mathbf{z}^{(c)}, \mathbf{y}\right)\right\| \leq C_{j} .
$$

If we have soft $*$-polynomial relations involving self-adjoint variables, we can replace each by two positive variables. A variable that is a contraction can be replaced by four positive variables. These replacements will preserve any homogeneity in a subset of the variables. Thus we can have a more flexible version of Theorem 3.1 . As stated, Theorem 3.2 is not a corollary as we are very specific in how the lifts are adjusted for the different types of variables.

Theorem 3.2. Suppose $p_{1}, \ldots, p_{J}$ are $N C$ *-polynomials in infinitely many variables that are homogeneous in the first $r$ variables, with each degree of homogeneity $d_{j}$ at least one. Suppose $S=\{1, \ldots, r\}$ is partitioned as

$$
S=S_{+} \cup S_{\mathrm{h}} \cup S_{\mathrm{g}},
$$

we have positive constants $C_{j}$, nonnegative constants $D_{k}, E_{k}, F_{k}$ and $G_{k}$, and consider the relations

$$
\begin{gathered}
0 \leq x_{k} \leq D_{k} \quad\left(k \in S_{+}\right) \\
-E_{k} \leq x_{k} \leq F_{k} \quad\left(k \in S_{\mathrm{h}}\right) \\
\left\|x_{k}\right\| \leq G_{k} \quad\left(k \in S_{\mathrm{g}}\right) \\
\left\|p_{j}(\mathbf{x}, \mathbf{y})\right\| \leq C_{j} .
\end{gathered}
$$

For every $C^{*}$-algebra $A$ and $I \triangleleft A$ an ideal, given $x_{1}, \ldots, x_{r}$ and $y_{1}, y_{2}, \ldots$ in $A$ so that $(\mathbf{x}, \mathbf{y})$ satisfies (3.7)-(3.9) and $(\pi(\mathbf{x}), \pi(\mathbf{y}))$ satisfies (3.10), there are elements $z_{1}, \ldots, z_{r}$ in $A$ so that $(\mathbf{z}, \mathbf{y})$ satisfy (3.7)-(3.10) and $\pi(\mathbf{z})=\pi(\mathbf{x})$. Moreover, it is possible to do so with

$$
\begin{aligned}
& z_{k}=\left(x_{k}\right)^{\frac{1}{2}} m^{2}\left(x_{k}\right)^{\frac{1}{2}} \quad\left(k \in S_{+}\right), \\
& z_{k}=m x_{k} m \quad\left(k \in S_{h}\right), \\
& z_{k}=x_{k} m^{2} \quad\left(k \in S_{\mathrm{g}}\right),
\end{aligned}
$$

for some $m$ in $1+I$ with $0 \leq m \leq 1$.

Proof. Let $\epsilon_{c}, \delta_{c}$, and $u_{\lambda}$ be as before. We modify the construction used for Theorem 3.1 by requiring $m_{0}=1$ and

$$
m_{c}=\left(1-\delta_{c} u_{\lambda_{c}}\right) m_{c-1}\left(1-\delta_{c} u_{\lambda_{c}}\right)
$$

and

$$
\begin{aligned}
& z_{k}^{(c)}=x_{k}^{\frac{1}{2}} m_{c}^{2} x_{k}^{\frac{1}{2}} \quad\left(k \in S_{+}\right), \\
& z_{k}^{(c)}=m_{c} x_{k} m_{c} \quad\left(k \in S_{h}\right), \\
& z_{k}^{(c)}=x_{k} m_{c}^{2} \quad\left(k \in S_{\mathrm{g}}\right) .
\end{aligned}
$$

We want

$$
\left\|p_{j}\left(\mathbf{z}^{(c)}, \mathbf{y}\right)\right\| \leq\left(1+\epsilon_{c}\right) C_{j},
$$

given that we have already defined $m_{c-1}$ in $1+I$ with

$$
\left\|p_{j}\left(\mathbf{z}^{(c-1)}, \mathbf{y}\right)\right\| \leq\left(1+\epsilon_{c-1}\right) C_{j} .
$$


To unify the initial step and subsequent steps, we take $\epsilon_{0}$ large enough to force (3.11) when $c=1$. We need to find the right $\lambda_{c}$ to define $z_{j}^{(c)}=w_{j}^{\left(\lambda_{c}\right)}$, where

$$
\begin{aligned}
& w_{k}^{(\lambda)}=x_{k}^{\frac{1}{2}}\left(\left(1-\delta_{c} u_{\lambda}\right) m_{c-1}\left(1-\delta_{c} u_{\lambda}\right)\right)^{2} x_{k}^{\frac{1}{2}} \quad\left(k \in S_{+}\right), \\
& w_{k}^{(\lambda)}=\left(\left(1-\delta_{c} u_{\lambda}\right) m_{c-1}\left(1-\delta_{c} u_{\lambda}\right)\right) x_{k}\left(\left(1-\delta_{c} u_{\lambda}\right) m_{c-1}\left(1-\delta_{c} u_{\lambda}\right)\right) \quad\left(k \in S_{h}\right), \\
& w_{k}^{(\lambda)}=x_{k}\left(\left(1-\delta_{c} u_{\lambda}\right) m_{c-1}\left(1-\delta_{c} u_{\lambda}\right)\right)^{2} \quad\left(k \in S_{\mathrm{g}}\right),
\end{aligned}
$$

and see

Therefore

$$
\lim _{\lambda}\left\|w_{j}^{(\lambda)}-\left(1-\delta_{c} u_{\lambda}\right)^{4} z_{j}^{(c-1)}\right\|=0
$$

$$
\begin{aligned}
\underset{\lambda}{\limsup \left\|p_{j}\left(\mathbf{w}^{(\lambda)}, \mathbf{y}\right)\right\|} & =\underset{\lambda}{\limsup }\left\|p_{j}\left(\left(1-\delta_{c} u_{\lambda}\right)^{4} \mathbf{z}^{(c-1)}, \mathbf{y}\right)\right\| \\
& =\underset{\lambda}{\limsup }\left\|p_{j}\left(\mathbf{z}^{(c-1)}, \mathbf{y}\right)\left(1-\delta_{c} u_{\lambda}\right)^{4 d_{j}}\right\| \\
& \leq \underset{\lambda}{\limsup }\left\|p_{j}\left(\mathbf{z}^{(c-1)}, \mathbf{y}\right)\left(1-\delta_{c} u_{\lambda}\right)\right\| \\
& \leq \max _{\lambda}\left(\left\|p_{j}(\pi(\mathbf{x}), \pi(\mathbf{y}))\right\|,\left(1-\delta_{c}\right)\left\|p_{j}\left(\mathbf{z}^{(c-1)}, \mathbf{y}\right)\right\|\right) \\
& \leq C_{j},
\end{aligned}
$$

and it is possible to choose the needed $\lambda_{c}$.

It is clear that $m_{c}$ stays in $1+I$, so these are all lifts of the original $\mathbf{x}$. What is left to check is that $m=\lim _{c} m_{c}$ exists. Indeed it does, as

$$
\begin{aligned}
\left\|m_{c}-m_{c-1}\right\| & =\left\|\left(1-\delta_{c} u_{\lambda_{c}}\right) m_{c-1}\left(1-\delta_{c} u_{\lambda_{c}}\right)-m_{c-1}\right\| \\
& \leq\left\|\left(\delta_{c} u_{\lambda_{c}}\right) m_{c-1}\left(1-\delta_{c} u_{\lambda_{c}}\right)\right\|+\left\|m_{c-1}\left(\delta_{c} u_{\lambda_{c}}\right)\right\| \\
& \leq 2\left\|\delta_{c} u_{\lambda_{c}} m_{c-1}\right\| \\
& \leq 2 \delta_{c} .
\end{aligned}
$$

From Theorem 3.2 we get a myriad of projective $C^{*}$-algebras, simply by adding relations such as $-1 \leq y_{j} \leq 1$ that are liftable and that impose a norm restriction forcing the universal $C^{*}$-algebra to exist. We generally add the relation $\left\|y_{j}\right\|=0$ to most of the $y_{j}$ so as to be working with a finitely generated projective $C^{*}$-algebra.

\section{Soft VERSIONS OF KNOWN PROJECTIVES}

Consider $C_{0}(X)$ where $X^{+}$is a (finite) tree. The presentation in [17] for the projective $C^{*}$-algebra $C_{0}(X)$ was based on a partial order $\preceq$ on $\{1, \ldots, s\}$. This was not a general partial order, it had to be the partial order on the nonroot vertices determined by paths away from the root. Let us call such a relation a tree order.

The presentation associated to the tree order $\preceq$ had generators $h_{1}, \ldots, h_{s}$ and relations

$$
\begin{aligned}
0 \leq h_{j} & \leq 1 \quad(j=1, \ldots, s), \\
h_{i} h_{j} & =h_{j} \quad(\text { if } i \prec j), \\
h_{i} h_{j} & =0 \quad(\text { if } i \npreceq j \text { and } j \npreceq i) .
\end{aligned}
$$


The last two lines of relations are not generally homogeneous in any subset of the variables. Taking advantage of the precise way the liftings are modified in our main theorem, we can still lift the soft version of these.

The concrete function in $C_{0}(X)$ that corresponds to the abstract $h_{j}$ only varies along one edge, the edge incident to the $j$ th vertex on a path from that vertex to the root. From this point of view, it makes more sense to index the generators by the edges (as in [17]) but in graph theory, tree orders are on the vertices.

We do need to make two changes to the relations. Some are redundant, since for positive elements

$$
h_{1} h_{2}=h_{2} \& h_{3} h_{1}=0 \Longrightarrow h_{3} h_{2}=0
$$

and

$$
h_{1} h_{2}=h_{2} \& h_{3} h_{1}=h_{1} \Longrightarrow h_{3} h_{2}=h_{2} .
$$

We adopt the usual terminology of $i$ being a child of $j$ whenever $j \preceq i$ and

$$
j \preceq k \preceq i \Longrightarrow k=j \text { or } k=i \text {. }
$$

The only relations we need are those that ask that the parent act as a unit on the child and that two children of the same parent must be orthogonal. We call two children of the same vertex siblings, of course. Children of children, and so forth, we call descendants.

A second change is that we replace $h_{i} h_{j}=h_{j}$ by

$$
\left(h_{i}-1\right) h_{j}\left(h_{i}-1\right)=0 .
$$

Theorem 4.1. Suppose $\preceq$ is a tree order on $\{1, \ldots, s\}$. The relations

$$
\begin{aligned}
0 \leq h_{j} \leq 1 \quad & (j=1, \ldots, s) \\
\left\|\left(h_{i}-1\right) h_{j}\left(h_{i}-1\right)\right\| \leq \epsilon \quad & (\text { if } j \text { is a child of } i) \\
\left\|h_{i} h_{j}\right\| & \leq \epsilon \quad(\text { if } i \text { and } j \text { are siblings })
\end{aligned}
$$

are liftable.

The following, slightly stronger result is more easily proven.

Theorem 4.2. Suppose $\preceq i s$ a tree order on $\{1, \ldots, s\}$ and $\epsilon>0$. For every $C^{*}$ algebra $A$ and $I \triangleleft A$ an ideal, given $h_{1}, \ldots, h_{s}$ in $A$ with $\mathbf{0} \leq \mathbf{h} \leq \mathbf{1}$ and

$$
\begin{aligned}
\left\|\pi\left(\left(h_{i}-1\right) h_{j}\left(h_{i}-1\right)\right)\right\| \leq \epsilon & (\text { if } j \text { is a child of } i), \\
\left\|\pi\left(h_{i}\right) \pi\left(h_{j}\right)\right\| \leq \epsilon & (\text { if } i \text { and } j \text { are siblings }),
\end{aligned}
$$

there are $k_{1}, \ldots, k_{s}$ in $A$ with $\mathbf{0} \leq \mathbf{k} \leq \mathbf{h}$ and $\pi(\mathbf{k})=\pi(\mathbf{h})$ and

$$
\begin{aligned}
\left\|\left(k_{i}-1\right) k_{j}\left(k_{i}-1\right)\right\| \leq \epsilon & (\text { if } j \text { is a child of } i) \\
\left\|k_{i} k_{j}\right\| \leq \epsilon & (\text { if } i \text { and } j \text { are siblings }) .
\end{aligned}
$$

Proof. The very trivial base case for our proof by induction is the case of zero generators.

Reindexing, we may assume the minimal elements (i.e., elders or vertices closest to the root) in this partial order are $\{1, \ldots, r\}$. If $m$ and $n$ are descendants of different minimal elements $i$ and $j$, then there are no relations involving both $h_{m}$ 
and $h_{n}$. The relations not involving the minimal elements are a disjoint union of relations of the type in the statement of the theorem.

The relations we need that involve the minimal elements are $0 \leq h_{j} \leq 1$ for $j=1, \ldots, r$ and

$$
\begin{aligned}
&\left\|\left(h_{i}-1\right) h_{m}\left(h_{i}-1\right)\right\| \leq \epsilon \quad(m \text { is the child of } i), \\
&\left\|k_{i} k_{j}\right\| \leq \epsilon \quad(1 \leq i<j \leq r) .
\end{aligned}
$$

These are homogeneous in $\left\{h_{1}, \ldots, h_{r}\right\}$. By Theorem 3.1 there are $k_{1}, \ldots, k_{r}$ in $A$ with $0 \leq k_{j} \leq h_{j}$ and $\pi\left(h_{j}\right)=\pi\left(h_{j}\right)$ for $j \leq r$ and

$$
\begin{aligned}
\left\|\left(k_{i}-1\right) h_{m}\left(k_{i}-1\right)\right\| & \leq \epsilon \quad(m \text { is the child of } i), \\
\left\|k_{i} k_{j}\right\| & \leq \epsilon \quad(1 \leq i<j \leq r) .
\end{aligned}
$$

The induction hypothesis tells us there are $k_{r+1}, \ldots, k_{s}$ with $0 \leq k_{m} \leq h_{m}$ and $\pi\left(k_{m}\right)=\pi\left(h_{m}\right)$ for $m>r$ with all the relations not involving indices $\{1, \ldots, r\}$. We might have lost the relations between some $h_{i}$ and $h_{m}$ with $m$ a child of $i$, but we have not, since

$$
\left(h_{i}-1\right) k_{m}\left(h_{i}-1\right) \leq\left(h_{i}-1\right) h_{m}\left(h_{i}-1\right) .
$$

A rather different example with a similar proof is a soft version of the projective $C^{*}$-algebra

$$
C^{*}\left\langle h, k, x \mid 0 \leq\left[\begin{array}{cc}
h k=0 \\
1-h & x^{*} \\
x & k
\end{array}\right] \leq 1\right\rangle
$$

considered in [19]. (For a detailed explanation of how the second relation is valid, see [20].)

Theorem 4.3. For any positive $\epsilon$, the $C^{*}$-algebra

$$
C^{*}\left\langle h, k, x \mid 0 \leq\left[\begin{array}{cc}
\|h k\| \leq \epsilon \\
1-h & x^{*} \\
x & k
\end{array}\right] \leq 1\right\rangle
$$

is projective.

Proof. Suppose $h, k$ and $x$ are in $A$, which we may assume is unital, such that

$$
\|\pi(h) \pi(k)\| \leq \epsilon
$$

and

$$
0 \leq\left[\begin{array}{cc}
1-\pi(h) & \pi(x)^{*} \\
\pi(x) & \pi(k)
\end{array}\right] \leq 1 .
$$

We know positive contractions lift to positive contractions from $\mathbf{M}_{2}(A / I)$ to $\mathbf{M}_{2}(A)$, and so we can find $\hat{h}, \hat{k}$, and $\hat{x}$ in $A$ so that $\pi(\hat{h})=\pi(h), \pi(\hat{k})=\pi(k), \pi(\hat{x})=$ $\pi(x)$ and

$$
0 \leq\left[\begin{array}{cc}
1-\hat{h} & \hat{x}^{*} \\
\hat{x} & \hat{k}
\end{array}\right] \leq 1
$$


The polynomial $h k$ is homogeneous in $k$ so Theorem 3.2 tells us there is $0 \leq m \leq 1$ in $1+I$ so that $\|\hat{h} m \hat{k} m\| \leq \epsilon$. Let $\bar{h}=\hat{h}, \bar{x}=m \hat{x}$ and $\bar{k}=m \hat{k} m$. These are still lifts of $h, x$ and $k$, and now $\|\bar{h} \bar{k}\| \leq \epsilon$ and

$$
\left[\begin{array}{cc}
1-\bar{h} & \bar{x}^{*} \\
\bar{h} & \bar{h}
\end{array}\right]=\left[\begin{array}{cc}
1 & 0 \\
0 & m
\end{array}\right]\left[\begin{array}{cc}
1-\hat{h} & \hat{x}^{*} \\
\hat{x} & \hat{k}
\end{array}\right]\left[\begin{array}{cc}
1 & 0 \\
0 & m
\end{array}\right]
$$

implies

$$
0 \leq\left[\begin{array}{cc}
1-\bar{h} & \bar{x}^{*} \\
\bar{h} & \bar{h}
\end{array}\right] \leq 1
$$

\section{Fattened Curves in various NC unit balls}

Theorem 5.1. Suppose $p_{1}, \ldots, p_{J}$ are $N C *$-polynomials in $x_{1}, \ldots, x_{s}$. Suppose $1 \leq r \leq s$ and each $p_{j}$ is homogeneous in $\left\{x_{1}, \ldots, x_{r}\right\}$ with degree of homogeneity $d_{j} \geq 1$. For $\epsilon>0$, the $C^{*}$-algebra

$$
A_{\epsilon}=C^{*}\left\langle\begin{array}{l|c}
x_{1}, \ldots, x_{s} & \begin{array}{c}
\left\|x_{k}\right\| \leq 1, k=1, \ldots, s \\
\left\|p_{j}\left(x_{1}, \ldots, x_{s}\right)\right\| \leq \epsilon, j=1, \ldots, J
\end{array}
\end{array}\right\rangle
$$

is projective.

Proof. This is an immediate consequence of Theorem 3.2 .

For a single $\mathrm{NC} *$-polynomial $p$, we can think of

$$
C^{*}\left\langle x_{1}, \ldots, x_{s} \mid \begin{array}{c}
\left\|x_{k}\right\| \leq 1 \\
\left\|p\left(x_{1}, \ldots, x_{s}\right)\right\| \leq \epsilon
\end{array}\right\rangle
$$

as an approximate zero locus of an $\mathrm{NC}$ curve in the $\mathrm{NC}$ unit square. Likewise, we can think of

$$
C^{*}\left\langle x_{1}, \ldots, x_{s}\right| \underset{\substack{\left\|p\left(x_{1}, \ldots, x_{s}\right)\right\| \leq \epsilon}}{\left\|\sum_{k=1}^{s} x_{k}^{*} x_{k}\right\| \leq 1} \|
$$

as an approximate zero locus of an $\mathrm{NC}$ curve in the $\mathrm{NC}$ unit ball. Notice that the "row contraction" condition $\left\|\sum x_{k}^{*} x_{k}\right\| \leq 1$ implies $\left\|x_{k}\right\| \leq 1$, so we can still apply Theorem 5.1. For $\epsilon>0$, and with $p$ homogeneous in $x_{1}, \ldots, x_{s}$, we find (5.1) and (5.2) define projective $C^{*}$-algebras.

We will see that it is possible to work with other unit balls, not just the ones corresponding to the $\ell^{2}$ - and $\ell^{\infty}$-norms.

Lemma 5.2. Suppose $0<\alpha<\infty$ is a scalar. For every $\epsilon>0$ there is a $\delta>0$ so that for any two positive contractions in any $C^{*}$-algebra,

$$
\|h k-k h\| \leq \delta \Longrightarrow\left\|(h k h)^{\alpha}-k^{\alpha} h^{2 \alpha}\right\| \leq \epsilon .
$$

Proof. This can be rephrased so it becomes a special case of Lemma 10 of [19], but it is easier to revise the proof. We know that for nonnegative scalars, $(x y x)^{\alpha}=y^{\alpha} x^{2 \alpha}$, so by spectral theory,

$$
h k=k h \Longrightarrow(h k h)^{\alpha}=k^{\alpha} h^{2 \alpha} .
$$


If the lemma is false, there must be some $\epsilon_{0}$ and $h_{n}$ and $k_{n}$ in $A_{n}$ with $0 \leq h_{n} \leq 1$ and $0 \leq k_{n} \leq 1$ and

and

$$
\left\|h_{n} k_{n}-k_{n} h_{n}\right\| \leq \frac{1}{n}
$$

This creates an element in

$$
\left\|\left(h_{n} k_{n} h_{n}\right)^{\alpha}-k_{n}^{\alpha} h_{n}^{2 \alpha}\right\| \geq \epsilon_{0} .
$$

$$
\prod A_{n} / \bigoplus A_{n}
$$

with $h k=k h$ and $(h k h)^{\alpha}-k^{\alpha} h^{2 \alpha} \neq 0$, a contradiction.

Theorem 5.3. Suppose $r$ is a natural number. For $0<p<\infty$, define

$$
B_{p}=C^{*}\left\langle x_{1}, \ldots, x_{r} \mid\left\|\sum_{k=1}^{r}\left(x_{k} x_{k}^{*}\right)^{\frac{p}{2}}\right\| \leq 1\right\rangle .
$$

If $0<p \leq \infty$, then $B_{p}$ is projective.

Proof. Suppose $I \triangleleft A$ with quasi-central approximate unit $u_{\lambda}$. Suppose $x_{k}$ are in $A$ with $\pi\left(x_{k}\right)$ in $A / I$ satisfying

$$
\left\|\sum_{k=1}^{r}\left(\pi\left(x_{k}\right)\left(\pi\left(x_{k}\right)\right)^{*}\right)^{\frac{p}{2}}\right\| \leq 1
$$

Let $z_{k}^{(0)}=x_{k}$, and let $\epsilon_{0}$ be sufficiently large so as to have

$$
\left\|\sum_{k=1}^{r}\left(z_{k}^{(0)}\left(z_{k}^{(0)}\right)^{*}\right)^{\frac{p}{2}}\right\| \leq 1+\epsilon_{0} .
$$

Choose $\delta_{c}$ a positive sequence decreasing to zero with $\delta_{1}=1$ and

$$
\sum_{c=1}^{\infty}\left(1-\left(1-\delta_{c}\right)^{\frac{1}{p}}\right)<\infty .
$$

Define $\epsilon_{c} \searrow 0$ for $c \geq 1$ by the formula

$$
\left(1-\delta_{c+1}\right)\left(1+\epsilon_{c}\right)=1 .
$$

Assume we have found $\mathbf{z}^{(c-1)}$ with

$$
\pi\left(\mathbf{z}^{(c-1)}\right)=\pi(\mathbf{x})
$$

and

$$
\left\|\sum_{k=1}^{r}\left(z_{k}^{(c-1)}\left(z_{k}^{(c-1)}\right)^{*}\right)^{\frac{p}{2}}\right\| \leq 1+\epsilon_{c} .
$$

Using Lemma 5.2 and Theorem 2.3 we find

$$
\begin{gathered}
\underset{\lambda}{\limsup }\left\|\sum_{k=1}^{r}\left(\left(1-\delta_{c} u_{\lambda}\right)^{\frac{1}{p}} z_{k}^{(c-1)}\left(z_{k}^{(c-1)}\right)^{*}\left(1-\delta_{c} u_{\lambda}\right)^{\frac{1}{p}}\right)^{\frac{p}{2}}\right\| \\
\quad=\limsup _{\lambda}\left\|\sum_{k=1}^{r}\left(z_{k}^{(c-1)}\left(z_{k}^{(c-1)}\right)^{*}\right)^{\frac{p}{2}}\left(1-\delta_{c} u_{\lambda}\right)\right\| \\
\leq 1 .
\end{gathered}
$$


We can choose $\lambda_{c}$ and set

$$
z_{k}^{(c)}=\left(1-\delta_{c} u_{\lambda_{c}}\right)^{\frac{1}{p}} z_{k}^{(c-1)},
$$

where $\lambda_{c}$ is large enough to ensure

$$
\left\|\sum_{k=1}^{r}\left(z_{k}^{(c)}\left(z_{k}^{(c)}\right)^{*}\right)^{\frac{p}{2}}\right\| \leq 1+\epsilon_{c} .
$$

The $z_{j}^{(c)}$ converge because

$$
\begin{aligned}
\left\|z_{k}^{(c)}-z_{k}^{(c-1)}\right\| & =\left\|\left(1-\delta_{c} u_{\lambda_{c}}\right)^{\frac{1}{p}} z_{k}^{(c-1)}-z_{k}^{(c-1)}\right\| \\
& \leq\left\|\left(1-\delta_{c} u_{\lambda_{c}}\right)^{\frac{1}{p}}-1\right\|\left\|z_{k}^{(c-1)}\right\| \\
& \leq\left(1-\left(1-\delta_{c}\right)^{\frac{1}{p}}\right)\left\|x_{k}\right\| .
\end{aligned}
$$

We still cannot tell if all the $B_{p}$ are isomorphic. They do interact with homogeneous $*$-polynomials in about the same fashion as the usual unit ball. The set of $\mathrm{NC} *$-polynomials that we know we can mix with the nonstandard unit ball condition depends on $p$. We have no idea if this is a limitation of our methods, or a real limitation.

Theorem 5.4. Suppose $r \leq s$ and $p_{1}, \ldots, p_{J}$ are $N C *$-polynomials in $x_{1}, \ldots, x_{s}$, each homogeneous in $\left\{x_{1}, \ldots, x_{r}\right\}$ with degree of homogeneity $d_{j}$ at least one. For $C_{j}>0$ and $0<q \leq 2$ the $C^{*}$-algebra

$$
A_{\epsilon}=C^{*}\left\langle x_{1}, \ldots, x_{s} \mid \begin{array}{c}
\left\|\sum_{k=1}^{s}\left(x_{k} x_{k}^{*}\right)^{\frac{q}{2}}\right\| \leq 1 \\
\left\|p_{j}\left(x_{1}, \ldots, x_{s}\right)\right\| \leq C_{j}, j=1, \ldots, J
\end{array}\right\rangle
$$

is projective.

Proof. Suppose we are given $\pi: A \rightarrow A / I$ with $x_{1}, \ldots, x_{s}$ in $A$ with

$$
\left\|\sum_{k=1}^{s}\left(\pi\left(x_{k} x_{k}^{*}\right)\right)^{\frac{q}{2}}\right\| \leq 1
$$

and

$$
\left\|p_{j}\left(\pi\left(x_{1}\right), \ldots, \pi\left(x_{s}\right)\right)\right\| \leq C_{j} .
$$

We first apply Theorem 5.3 to find $y_{1}, \ldots, y_{s}$ in $A$ with $\pi\left(y_{k}\right)=\pi\left(x_{k}\right)$ and

$$
\left\|\sum_{k=1}^{s}\left(y_{k} y_{k}^{*}\right)^{\frac{q}{2}}\right\| \leq 1
$$

Theorem 3.2 gives us $z_{1}, \ldots, z_{s}$ in $A$ with $\pi\left(z_{j}\right)=\pi\left(x_{j}\right)$ and

$$
\left\|p_{j}\left(z_{1}, \ldots, z_{s}\right)\right\| \leq C_{j}
$$

but also with $z_{k}=y_{k} m$ for $k \leq r$ and $z_{k}=y_{k}$ for $k>r$, where $0 \leq m \leq 1$. Therefore

$$
z_{k} z_{k}^{*}=y_{k} m^{2} y_{k}^{*} \leq y_{k} y_{k}^{*}
$$


for $1 \leq k \leq r$. Since for $q \leq 2$ the function $t^{q / 2}$ is operator-monotone, we get

$$
\sum_{k=1}^{s}\left(z_{k} z_{k}^{*}\right)^{\frac{q}{2}} \leq \sum_{k=1}^{s}\left(y_{k} y_{k}^{*}\right)^{\frac{q}{2}} \leq 1 .
$$

Theorem 5.5. Suppose $p_{1}, \ldots, p_{J}$ are homogeneous, degree- $d_{j} N C *$-polynomials in $x_{1}, \ldots, x_{r}$ with $d_{j} \geq 1$. For $C_{j}>0$ and $2<q<\infty$, the $C^{*}$-algebra

$$
A_{\epsilon}=C^{*}\left\langle x_{1}, \ldots, x_{r} \mid \begin{array}{c}
\left\|\sum_{k=1}^{r}\left(x_{k} x_{k}^{*}\right)^{\frac{q}{2}}\right\| \leq 1 \\
\left\|p_{j}\left(x_{1}, \ldots, x_{r}\right)\right\| \leq C_{j}, j=1, \ldots, J
\end{array}\right\rangle
$$

is projective.

Proof. Suppose we are given $\pi: A \rightarrow A / I$ with $x_{1}, \ldots, x_{r}$ in $A$ with

$$
\left\|\sum_{k=1}^{r}\left(\pi\left(x_{k} x_{k}^{*}\right)\right)^{\frac{q}{2}}\right\| \leq 1
$$

and

$$
\left\|p_{j}\left(\pi\left(x_{1}\right), \ldots, \pi\left(x_{r}\right)\right)\right\| \leq C_{j} .
$$

Choose $\delta_{c}$ and $\epsilon_{c}$ as before, with the $\delta_{c}$ summable. Keeping with our earlier notation, we are going to define $\mathbf{z}^{(c)}$ from $\mathbf{z}^{(c-1)}$ by

$$
z_{k}^{(c)}=\left(1-\delta_{c} u_{\lambda_{c}}\right) z_{k}^{(c-1)} .
$$

Lemma 5.2 and Theorem 2.3 give us

$$
\begin{aligned}
\underset{\lambda}{\limsup }\left\|\sum_{k=1}^{r}\left(\left(1-\delta_{c} u_{\lambda}\right) z_{k}^{(c-1)}\left(z_{k}^{(c-1)}\right)^{*}\left(1-\delta_{c} u_{\lambda}\right)\right)^{\frac{q}{2}}\right\| \\
=\underset{\lambda}{\limsup }\left\|\sum_{k=1}^{r}\left(z_{k}^{(c-1)}\left(z_{k}^{(c-1)}\right)^{*}\right)^{\frac{q}{2}}\left(1-\delta_{c} u_{\lambda}\right)^{q}\right\| \\
\leq \underset{\lambda}{\limsup }\left\|\sum_{k=1}^{r}\left(z_{k}^{(c-1)}\left(z_{k}^{(c-1)}\right)^{*}\right)^{\frac{q}{2}}\left(1-\delta_{c} u_{\lambda}\right)\right\| \\
=1
\end{aligned}
$$

and

$$
\begin{aligned}
\underset{\lambda}{\limsup } \| p_{j}((1- & \left.\left.\delta_{1} u_{\lambda}\right) \mathbf{z}^{(c-1)}\right) \| \\
& =\limsup _{\lambda}\left\|p_{j}\left(\mathbf{z}^{(c-1)}\right)\left(1-\delta_{c} u_{\lambda}\right)^{d_{j}}\right\| \\
& \leq \limsup _{\lambda}\left\|p_{j}\left(\mathbf{z}^{(c-1)}\right)\left(1-\delta_{c} u_{\lambda}\right)\right\| \\
& =C_{j} .
\end{aligned}
$$


The limit of the $z_{j}^{(c)}$ will exist because

$$
\begin{aligned}
\left\|z_{k}^{(c)}-z_{k}^{(c-1)}\right\| & =\left\|\left(1-\delta_{c} u_{\lambda_{c}}\right) z_{k}^{(c-1)}-z_{k}^{(c-1)}\right\| \\
& \leq\left\|\left(1-\delta_{c} u_{\lambda_{c}}\right)-1\right\|\left\|z_{k}^{(c-1)}\right\| \\
& \leq \delta_{c}\left\|x_{k}\right\| .
\end{aligned}
$$

\section{SOFT CYLINDERS}

When we stray from homogeneous relations, we come across $K$-theoretical obstructions to projectivity. To illustrate what properties can still hold, we offer the example of the "soft cylinder". The weaker properties are semiprojectivity (as in [4) and the residually finite dimensional (RFD) property. Projectivity implies semiprojectivity and also RFD ([22, §1]).

For $\epsilon \geq 0$ we define the soft cylinder almost like Exel's soft torus ([14]):

$$
A_{\epsilon}=C_{1}^{*}\left\langle u, h \mid u^{*} u=u u^{*}=1,-1 \leq h \leq 1,\|u h-h u\| \leq \epsilon\right\rangle .
$$

Notice we retained some homogeneity.

Theorem 6.1. For positive $\epsilon$, the soft cylinder $A_{\epsilon}$ is semiprojective.

Proof. Suppose $B$ is a unital $C^{*}$-algebra, with ideal $I=\overline{\bigcup I}_{n}$ for some increasing sequence of ideals $I_{n}$. Suppose we are given $u$ and $h$ in $B / I$ where $u$ is unitary, $-1 \leq h \leq 1$, and

$$
\|u h-h u\| \leq \epsilon .
$$

For some $n$ it is possible to lift $u$ to $v$ in $B / I_{n}$ that is a unitary (4, Prop. 2.21]). Take any lift of $h$ to $-1 \leq k \leq 1$ in $B / I_{n}$. Theorem 3.2 tells us there is $\hat{k}$ in $A / I$ with $-1 \leq \hat{k} \leq 1$ and

$$
\|v \hat{k}-\hat{k} v\| \leq \epsilon .
$$

Eilers and Exel ([1]) have shown that the soft torus is RFD. The same can be said, and proven much more easily, for the soft cylinder.

Theorem 6.2. For positive $\epsilon$, the soft cylinder $A_{\epsilon}$ is RFD.

Proof. Consider the surjection

$$
\rho: C\left(S^{1}\right) *_{\mathbb{C}} C[0,1] \rightarrow \widetilde{A_{\epsilon}}
$$

that sends the obvious unitary generator to $u$ and the obvious positive, norm-1 generator to $h$. By [15, Theorem 3.2] the free product is RFD. Theorem 3.2 tells us that $\rho$ is split. Thus $A_{\epsilon}$ can be embedded in an RFD $C^{*}$-algebra and so is itself RFD.

Our lifting theorems can be used to determine whether many more $C^{*}$-algebras are RFD. The study of weak projectivity ([21]) and RFD of the $C^{*}$-algebras associated to rather general relations that have some homogeneity might lead to some interesting examples. 


\section{Cones ARE Limits of PROJECTIVE $C^{*}$-Algebras}

We end with a tantalizing result: every cone is the limit of projectives. As a $C^{*}$-algebra with a projective cone must be semiprojective ([5, II.8.3.10]) it would seem that we are close to proving that every separable $C^{*}$-algebra is a limit of semiprojective $C^{*}$-algebras.

We say definitively, projectivity is not "extremely rare", cf. [18, p. 73].

Lemma 7.1. Suppose $A$ is the unital $C^{*}$-algebra

$$
A=C_{1}^{*}\left\langle x_{1}, x_{2}, \ldots \mid \begin{array}{cc}
-C_{k} \leq x_{k} \leq C_{k} \quad(\forall k) \\
p_{j}(\mathbf{x})=0 \quad(\forall j)
\end{array}\right\rangle,
$$

where the $p_{1}, p_{2} \cdots$ are $N C$ polynomials in the $x_{k}$ of degrees $D_{j}$ with zero constant term. Then the cone $\mathbf{C} A$ has presentation

$$
\mathbf{C} A=C^{*}\left\langle h, x_{1}, x_{2}, \ldots \mid \begin{array}{c}
0 \leq h \leq 1 \\
h x_{k}=x_{k} h \quad(\forall k) \\
-C_{k} h \leq x_{k} \leq C_{k} h \quad(\forall k) \\
q_{j}(h, \mathbf{x})=0 \quad(\forall j)
\end{array}\right\rangle,
$$

where $q_{j}$ is the NC polynomial derived from the $p_{j}$ by padding monomials on the left with various powers of $h$ so that $q_{j}$ is homogeneous with degree $D_{j}$.

Proof. To illustrate the construction of the $q_{j}$, if

$$
p_{1}=x_{1}+3 x_{1} x_{2}^{*} x_{1}
$$

then

$$
q_{1}=h^{2} x_{1}+3 x_{1} x_{2}^{*} x_{1}
$$

In general, we can break up $p_{j}$ into homogeneous summands

$$
p_{j}=\sum_{d=1}^{D_{j}} p_{j, d}
$$

and then describe the $q_{j}$ as

$$
q_{j}(h, \mathbf{x})=\sum_{d=1}^{D_{j}} h^{D_{j}-d} p_{j, d}(\mathbf{x}) .
$$

Let the universal unital $C^{*}$-algebra for these relations be denoted $\mathcal{U}$. This exists, as the relations satisfy the needed four axioms as in $[20$. One of the axioms is that setting all variables to the zero elements in $\{0\}$ leads to a representation of the relations, which is true because we require the constant terms to be zero.

To define a $*$-homomorphism $\mathcal{U} \rightarrow \mathbf{C} A$, we define in $\mathbf{C} A=C_{0}((0,1], A)$ elements $\tilde{x}_{k}=t x_{k}$ and $\tilde{h}=t$, shorthand for $\tilde{x}(t)=t x$ and so forth. It is obvious that $0 \leq \tilde{h} \leq 1$ and that $\tilde{h}$ commutes with each $\tilde{x}_{k}$. Also

$$
-C_{k} \leq x_{k} \leq C_{k} \Longrightarrow-t C_{k} \leq t x_{k} \leq t C_{k}
$$


so $-C_{j} \tilde{h} \leq \tilde{x}_{j} \leq C_{j} \tilde{h}$. The last relation holds as well since

$$
\begin{aligned}
\left(q_{j}(\tilde{h}, \tilde{\mathbf{x}})\right)(t) & =\sum_{d=1}^{D_{j}} t^{D_{j}-d} p_{j, d}(t \mathbf{x}) \\
& =t^{D_{j}} \sum_{d=1}^{D_{j}} p_{j, d}(\mathbf{x}) \\
& =t^{D_{j}} p_{j}(\mathbf{x}) \\
& =0 .
\end{aligned}
$$

Next we will show this map is onto. Basic algebra and the usual isomorphism of $\mathbf{C} A$ with $C_{0}(0,1] \otimes A$ tells us that functions of the form $t^{m} w$ generate the cone, where $w$ ranges over words in the $x_{k}$. Suppose $w=w_{1} w_{2} \cdots w_{n}$. If $m \geq n$, then this is easily in the image, as

$$
t^{m} w=t^{m-n}\left(t w_{1}\right)\left(t w_{2}\right) \cdots\left(t w_{n}\right) .
$$

If $1 \leq m<n$, then the Stone-Weierstrass theorem tells us we can approximate in $C_{0}(0,1]$ the function $t^{m}$ by a polynomial in $t^{n}, t^{n+1}, \ldots$, and so we can approximate $t^{m} w$ by a polynomial in $t^{n} w, t^{n+1} w, \ldots$ and the map is indeed onto. We turn to proving it is one-to-one.

Consider an irreducible representation in $\mathbb{B}(\mathbb{H})$ of the relations defining $\mathcal{U}$ by $H$ and $X_{1}, X_{2}, \ldots$. Since $0 \leq H \leq 1$ and $H X_{k}=X_{k} H$, we find that $H$ is central and so $H=\lambda I$ for some scalar $\lambda$ with $0 \leq \lambda \leq 1$. If $\lambda=0$, then $H=0$ and

$$
-C_{k} \lambda \leq X_{k} \leq C_{k} \lambda \Longrightarrow X_{k}=0
$$

This is the zero representation, which is the pullback of the zero representation of $\mathrm{C} A$. If $\lambda$ is positive, then

$$
-C_{k} \lambda \leq X_{k} \leq C_{k} \lambda \Longrightarrow-C_{k} \leq \lambda^{-1} X_{k} \leq C_{k},
$$

and $q_{j}(H, \mathbf{X})=0$ implies

$$
\begin{aligned}
p_{j}\left(\lambda^{-1} \mathbf{X}\right) & =\sum_{d=1}^{D_{j}} \lambda^{-d} p_{j, d}(\mathbf{X}) \\
& =\lambda^{-D_{j}} \sum_{d=1}^{D_{j}} H^{D_{j}-d} p_{j, d}(\mathbf{X}) \\
& =\lambda^{-D_{j}} q_{j}(H, \mathbf{X}) \\
& =0
\end{aligned}
$$

Thus the $\lambda^{-1} X_{k}$ form a representation of $A$ on $\mathbb{H}$ and so a representation of $\mathbf{C} A$ via the composition

$$
\mathbf{C} A \stackrel{\delta_{\lambda}}{\longrightarrow} A \longrightarrow \mathbb{B}(\mathbb{H}) \text {. }
$$

This sends $\tilde{h}$ to $\lambda I=H$ and $\tilde{x}_{k}$ to $X_{k}$, finishing the proof.

Theorem 7.2. If $q_{1}, q_{2} \ldots$ are homogeneous $N C$ polynomials, each of degree at least one, in noncommuting variables $h, x_{1}, x_{2}, \ldots$, then for positive constants $C_{1}, \ldots, C_{J}$ 
and $D_{1}, \ldots, D_{K}$, the $C^{*}$-algebra

$$
C^{*}\left\langle h, x_{1}, x_{2}, \ldots \mid \begin{array}{c}
0 \leq h \leq 1, \\
-C_{k} h \leq x_{k} \leq C_{k} h, \quad(1 \leq k \leq K) \\
\left\|q_{j}\left(h, x_{1}, x_{2}, \ldots\right)\right\| \leq D_{j} \quad(1 \leq j \leq J)
\end{array}\right\rangle
$$

is projective.

Proof. We can find some $r$ so that $x_{r+1}, x_{r+2}, \ldots$ are not in any of the polynomials $q_{1}, \ldots, q_{J}$. If we relabel these $y_{1}, y_{2}, \ldots$, our lifting problem becomes

$$
\begin{gathered}
0 \leq h \leq 1, \\
-C_{k} h \leq x_{k} \leq C_{k} h, \\
-C_{k}^{\prime} h \leq y_{k} \leq C_{k}^{\prime} h, \\
\left\|q_{j}(h, \mathbf{x})\right\| \leq D_{j} \quad(1 \leq j \leq J),
\end{gathered}
$$

where now the $q_{j}$ are homogeneous in $\left\{h, x_{1}, \ldots, x_{r}\right\}$. We are using $\mathbf{x}$ for $\left(x_{1}, \ldots, x_{r}\right)$.

Given $h, x_{k}$ and $y_{k}$ in $A$ with

$$
\begin{gathered}
0 \leq \pi(h) \leq 1, \\
-C_{k} \pi(h) \leq \pi\left(x_{k}\right) \leq C_{k} \pi(h), \\
-C_{k}^{\prime} \pi(h) \leq \pi\left(y_{k}\right) \leq C_{k}^{\prime} \pi(h), \\
\left\|q_{j}(\pi(h), \pi(\mathbf{x}))\right\| \leq D_{j} \quad(1 \leq j \leq J),
\end{gathered}
$$

we first find a new lift $\hat{h}$ of $\pi(h)$ with

$$
0 \leq \hat{h} \leq 1
$$

Using [8, Corollary 2.2] we find $\hat{x}_{k}$ and $\hat{y}_{k}$ with

$$
\begin{aligned}
& -C_{k} \hat{h} \leq \hat{x}_{k} \leq C_{k} \hat{h}, \\
& -C_{k}^{\prime} \hat{h} \leq \hat{y}_{k} \leq C_{k}^{\prime} \hat{h}
\end{aligned}
$$

and $\pi\left(\hat{x}_{k}\right)=\pi\left(x_{j}\right)$ and $\pi\left(\hat{y}_{k}\right)=\pi\left(y_{j}\right)$. By Theorem 3.2 there is an $m$ with $0 \leq m \leq 1$ in $1+I$ so that

$$
\left\|q_{j}(m \hat{h} m, m \hat{\mathbf{x}} m)\right\| \leq D_{j} .
$$

Our desired lifts are $m \hat{h} m, m \hat{\mathbf{x}} m$ and $m \hat{\mathbf{y}} m$.

Lemma 7.3. Let $D$ be a separable $C^{*}$-algebra. Then

$$
D \cong C^{*}\left\langle x_{1}, x_{2}, \ldots \mid \begin{array}{cc}
-C_{j} \leq x_{j} \leq C_{j} & (\forall j) \\
p_{k}\left(x_{1}, x_{2}, \ldots\right)=0 & (\forall k)
\end{array}\right\rangle
$$

for a countable collection of NC polynomials.

Proof. Example 1.3(b) in 4 tells us that $D$ has a presentation with countably many generators, countably many relations in the form of an $\mathrm{NC} *$-polynomial set to zero and countably many norm conditions. We will modify Blackadar's method a bit.

Let $\mathbb{F}=\mathbb{Q}+i \mathbb{Q}$, which is a countable dense subfield of $\mathbb{C}$. Select a countable dense sequence in $D$ and apply to this sequence all polynomials over $\mathbb{F}$ in countably many variables. This results in a countable, dense $\mathbb{F}$-*-subalgebra $B$ of $D$. Enumerate $B$ as $x_{1}, x_{2}, \ldots$ The algebraic operations for $B$ can be encoded in $*$-polynomial 
relations. For example, if $\alpha x_{j}=x_{k}$ for some $\alpha$ in $\mathbb{F}$, then we use the relation $\alpha x_{j}-x_{k}=0$. If $x_{j}^{*}=x_{k}$, then we use the relation $x_{j}^{*}-x_{k}=0$, and so forth. This means $B$ is the universal $\mathbb{F}$-*-algebra for generators $x_{1}, x_{2}, \ldots$ and some countable set of $*$-polynomial relations $p_{j}\left(x_{1}, x_{2}, \ldots\right)=0$. We now add to these relations the $C^{*}$-relations $\left\|x_{k}\right\| \leq C_{k}$ where $C_{k}$ is the norm of the element $x_{k}$ in $D$. Then any function $f: B \rightarrow G$, for $G$ a $C^{*}$-algebra, that satisfies these relations is first of all an $\mathbb{F}$-linear $*$-algebra homomorphism. It is continuous with respect to the norm on $D$ since $x_{j}-x_{k}$ will equal some $x_{\ell}$ so we have the relation $\left\|f\left(x_{\ell}\right)\right\| \leq\left\|x_{\ell}\right\|$ and so

$$
\left\|f\left(x_{j}\right)-f\left(x_{k}\right)\right\|=\left\|f\left(x_{\ell}\right)\right\| \leq\left\|x_{\ell}\right\|=\left\|x_{j}-x_{k}\right\| .
$$

It therefore extends to a continuous function $\varphi: D \rightarrow G$. This extended function will be linear over $\mathbb{C}$. To verify this, consider $\alpha=\lim \alpha_{n}$, a limit of scalars from $\mathbb{F}$, and $d=\lim d_{j}$, a limit of elements in $B$. Then

$$
\varphi\left(\alpha_{n} d\right)=\varphi\left(\lim _{j} \alpha_{n} d_{j}\right)=\lim _{j} f\left(\alpha_{n} d_{j}\right)=\alpha_{n} \lim _{j} f\left(d_{j}\right)=\alpha_{n} \varphi(d)
$$

and

$$
\varphi(\alpha d)=\varphi\left(\lim _{n} \alpha_{n} d\right)=\lim _{n} \varphi\left(\alpha_{n} d\right)=\lim _{n} \alpha_{n} \varphi(d)=\alpha \varphi(d) .
$$

Finally, continuity implies that $\varphi$ is a $*$-homomorphism. It is uniquely determined by $f$ and so $D$ is universal for these relations.

We can eliminate many of the norm conditions. Suppose we keep only the norm restrictions $\left\|x_{k}\right\| \leq C_{k}$ for those $x_{k}$ that are self-adjoint. Then the estimate that gave continuity changes a little. Any $x_{j}-x_{k}$ will equal some $x_{\ell}$ and for some $r$ and $s$ we will have $x_{r}=\frac{1}{2} x_{\ell}+\frac{1}{2} x_{\ell}^{*}$ and $x_{s}=\frac{-i}{2} x_{\ell}-\frac{i}{2} x_{\ell}^{*}$. Of course these are the Hermitian and anti-Hermitian parts of $x_{\ell}$, and as they are self-adjoint we have the relations $\left\|f\left(x_{r}\right)\right\| \leq\left\|x_{r}\right\|$ and $\left\|f\left(x_{s}\right)\right\| \leq\left\|x_{s}\right\|$. Therefore

$$
\begin{aligned}
\left\|f\left(x_{j}\right)-f\left(x_{k}\right)\right\| & =\left\|f\left(x_{r}\right)+i f\left(x_{s}\right)\right\| \\
& \leq\left\|f\left(x_{r}\right)\right\|+\left\|f\left(x_{s}\right)\right\| \\
& \leq\left\|x_{r}\right\|+\left\|x_{s}\right\| \\
& \leq 2\left\|x_{\ell}\right\| \\
& =2\left\|x_{j}-x_{k}\right\| .
\end{aligned}
$$

This still gives us continuity and so the rest of the proof goes through.

We can toss the generators that are not self-adjoint if we modify each polynomial by the evaluating $x_{k}$ at $x_{r}+i x_{s}$ whenever $x_{r}$ and $x_{s}$ are the real and imaginary parts of $x_{k}$. Among the polynomial relations will be $x_{j}^{*}-x_{j}=0$ for the generators we are keeping. Given this, it is our option to use the relation $\left\|x_{j}\right\| \leq C_{j}$ or $-C_{j} \leq x_{j} \leq C_{j}$.

Theorem 7.4. If $A$ is a separable $C^{*}$-algebra, then its cone $\mathbf{C} A$ is isomorphic to the inductive limit of a countable system of projective $C^{*}$-algebras with surjective bonding maps.

Proof. We start with the case where $A=\widetilde{D}$ for some separable, possibly unital $C^{*}$-algebra.

Lemma 7.3 tells us

$$
A \cong C_{1}^{*}\left\langle x_{1}, x_{2}, \ldots \mid \begin{array}{cc}
-C_{k} \leq x_{k} \leq C_{k} & (\forall k) \\
p_{j}\left(x_{1}, x_{2}, \ldots\right)=0 & (\forall j)
\end{array}\right\rangle,
$$


and then Lemma 7.1 tells us

$$
\mathbf{C} A \cong C^{*}\left\langle h, x_{1}, x_{2}, \ldots \mid \begin{array}{c}
0 \leq h \leq 1 \\
h x_{k}=x_{k} h \quad(\forall k) \\
-C_{k} h \leq x_{k} \leq C_{k} h \quad(\forall k) \\
q_{j}\left(h, x_{1}, x_{2}, \ldots\right)=0
\end{array}\right\rangle,
$$

where the $q_{k}$ are homogeneous. Clearly,

$$
\mathbf{C} A \cong \lim _{\longrightarrow} P_{n}
$$

where

$$
P_{n}=C^{*}\left\langle h, x_{1}, x_{2}, \ldots \mid \begin{array}{c}
0 \leq h \leq 1 \\
-C_{k} h \leq x_{k} \leq C_{k} h, \quad(\forall k) \\
\left\|h x_{k}-x_{k} h\right\| \leq \frac{1}{n}, \quad(k=1, \ldots, n) \\
\left\|q_{j}\left(x_{1}, x_{2}, \ldots\right)\right\| \leq \frac{1}{n} \quad(j=1, \ldots, n)
\end{array}\right\rangle .
$$

Since the commutators are homogeneous NC polynomials, Theorem 7.2 applies and the $P_{n}$ are projective. We are done for $\mathbf{C} A=\mathbf{C}(\widetilde{D})$. What about $\mathbf{C} D$ ?

We have the exact sequence

$$
0 \longrightarrow \mathbf{C} D \longrightarrow \mathbf{C} A \longrightarrow \mathbf{C} C \longrightarrow 0 \text {. }
$$

Of course $\mathbf{C} \mathbb{C}$ equals $C_{0}(0,1]$ and is projective. Let $Q_{n}$ be the kernel of the map of $P_{n}$ onto $C_{0}(0,1]$ that sends $h$ to $t \mapsto t$ and $x_{k}$ to zero. Then we have

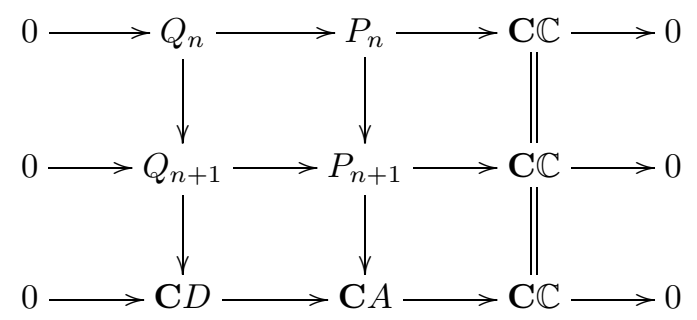

with the rows exact. Also, $\mathbf{C} D$ is isomorphic to $\underset{\lim }{\longrightarrow} Q_{n}$, which we can see as follows.

There is a $*$-homomorphism

$$
\varphi: \lim _{\longrightarrow} Q_{n} \rightarrow \mathbf{C} D
$$

induced by the maps $Q_{n} \rightarrow \mathbf{C} D$. The maps $Q_{n} \rightarrow P_{n}$ are inclusions and hence isometries. Theorem 13.1.2.2 in [18] implies that the induced map $\underset{\lim }{\longrightarrow} Q_{n} \rightarrow \underset{\lim }{\longrightarrow} P_{n}$ is an also an isometry. From the commutative diagram

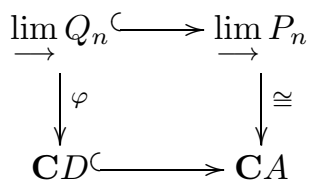


we conclude $\varphi$ is injective. As to surjectivity, consider $x$ in $\mathbf{C} D$. This gets sent to zero in $\mathbf{C} \mathbb{C}$. Any lift of $x$ to $y$ in $P_{1}$ is also sent to zero in $\mathbf{C} \mathbb{C}$, and so is $Q_{n}$. This shows $\varphi$ is onto.

By Theorem 5.3 of 22$]$ the $Q_{n}$ are projective.

\section{REFERENCES}

[1] Charles A. Akemann, Left ideal structure of $C^{*}$-algebras, J. Functional Analysis 6 (1970), 305-317. MR0275177 (43:934)

[2] Charles A. Akemann and Gert K. Pedersen, Ideal perturbations of elements in $C^{*}$-algebras, Math. Scand. 41 (1977), no. 1, 117-139. MR.0473848 (57:13507)

[3] William Arveson, Subalgebras of $C^{*}$-algebras. III. Multivariable operator theory, Acta Math. 181 (1998), no. 2, 159-228. MR1668582 (2000e:47013)

[4] Bruce Blackadar, Shape theory for $C^{*}$-algebras, Math. Scand. 56 (1985), no. 2, 249-275. MR813640 (87b:46074)

[5] - Operator algebras, Encyclopaedia of Mathematical Sciences, vol. 122, SpringerVerlag, Berlin, 2006, Theory of $C^{*}$-algebras and von Neumann algebras, Operator Algebras and Non-commutative Geometry, III. MR2188261 (2006k:46082)

[6] Karol Borsuk, Theory of shape, PWN-Polish Scientific Publishers, Warsaw, 1975, Monografie Matematyczne, Tom 59. MR0418088 (54:6132)

[7] A. Chigogidze and AN Dranishnikov, Which compacta are noncommutative ARs?, Topology and its Applications 157 (2010), no. 4, 774-778.

[8] Kenneth R. Davidson, Lifting positive elements in $C^{*}$-algebras, Integral Equations Operator Theory 14 (1991), no. 2, 183-191. MR 1090700 (92f:46065)

[9] Kenneth R. Davidson and David R. Pitts, Nevanlinna-Pick interpolation for noncommutative analytic Toeplitz algebras, Integral Equations Operator Theory 31 (1998), no. 3, 321-337. MR.1627901 (2000g:47016)

[10] Edward G. Effros and Jerome A. Kaminker, Homotopy continuity and shape theory for $C^{*}$ algebras, Geometric methods in operator algebras (Kyoto, 1983), Pitman Res. Notes Math. Ser., vol. 123, Longman Sci. Tech., Harlow, 1986, pp. 152-180. MR866493 (88a:46082)

[11] Søren Eilers and Ruy Exel, Finite-dimensional representations of the soft torus, Proc. Amer. Math. Soc. 130 (2002), no. 3, 727-731 (electronic). MR1866027 (2002i:46051)

[12] Søren Eilers and Terry A. Loring, Computing contingencies for stable relations, Internat. J. Math. 10 (1999), no. 3, 301-326. MR1688149 (2000d:46084)

[13] Søren Eilers, Terry A. Loring, and Gert K. Pedersen, Stability of anticommutation relations: an application of noncommutative $C W$ complexes, J. Reine Angew. Math. 499 (1998), 101143. MR1631120 (99e:46067)

[14] Ruy Exel, The soft torus and applications to almost commuting matrices, Pacific J. Math. 160 (1993), no. 2, 207-217. MR1233352 (94f:46091)

[15] Ruy Exel and Terry A. Loring, Finite-dimensional representations of free product $C^{*}$ algebras, Internat. J. Math. 3 (1992), no. 4, 469-476. MR1168356 (93f:46091)

[16] Stanislaw Lojasiewicz, Triangulation of semi-analytic sets, Ann. Scuola Norm. Sup. Pisa (3) 18 (1964), 449-474. MR0173265 (30:3478)

[17] Terry A. Loring, Projective $C^{*}$-algebras, Math. Scand. 73 (1993), no. 2, 274-280. MR 1269264 (95h:46085)

[18] , Lifting solutions to perturbing problems in $C^{*}$-algebras, Fields Institute Monographs, vol. 8, American Mathematical Society, Providence, RI, 1997. MR1420863 (98a:46090)

[19] _ A projective $C^{*}$-algebra related to K-theory, J. Funct. Anal. 254 (2008), no. 12, 3079-3092. MR 2418619 (2009g:46136)

[20] _ $C^{*}$-algebra relations, Math. Scand. 107, no. 1, 43-72. MR2679392

[21] _ Weakly projective $C^{*}$-algebras, Rocky Mountain J. Math. (to appear), http://arxiv.org/abs/0905.1520.

[22] Terry A. Loring and Gert K. Pedersen, Projectivity, transitivity and AF-telescopes, Trans. Amer. Math. Soc. 350 (1998), no. 11, 4313-4339. MR1616003 (99c:46065)

[23] Catherine L. Olsen and Gert K. Pedersen, Corona $C^{*}$-algebras and their applications to lifting problems, Math. Scand. 64 (1989), no. 1, 63-86. MR.1036429 (91g:46064) 
[24] Tatiana Shulman, Lifting of nilpotent contractions, Bull. London Math. Soc. 40 (2008), no. 6, 1002-1006. MR 2471949 (2009k:46102)

[25] Viktor A. Vassiliev, Applied Picard-Lefschetz theory, Mathematical Surveys and Monographs, vol. 97, American Mathematical Society, Providence, RI, 2002. MR.1930577 (2003k:32043)

Department of Mathematics and Statistics, University of New Mexico, Albuquerque, New MeXICO 87131

Department of Mathematics, University of Copenhagen, Universitetsparken 5, DK2100 Copenhagen $\varnothing$, Denmark

Current address: Department of Mathematics, Siena College, 515 Loudon Road, Loudonville, New York 12211 